journal club

\title{
Das Alzheimer-Peptid Abeta und Schlafstörungen bilden einen Teufelskreis
}

Fragestellung: Wie beeinflusst die lokale Ablagerung von AbetaPeptiden Nicht-REM-Schlaf und Gedächtniskonsolidierung?

Hintergrund: Es ist seit langem bekannt, dass Schlaf die Gesundheit des Menschen allgemein, aber auch seine Gedächtnisleistung und Lernfähigkeit beeinflusst. Mehrere unabhängige Untersuchungen legen derzeit nahe, dass es zudem einen unmittelbaren Zusammenhang zwischen Alzheimer-Pathologie und Schlafqualität gibt. So sind zum Beispiel die langsamen Hirnwellen des Nicht-REM-Schlafs (NREM-slow wave activity; NREM-SWA) bei älteren Erwachsenen reduziert und assoziiert mit der Gedächtnisleistung [1]. Gleichzeitig korreliert die kortikale Menge an Abeta-Peptiden mit einer subjektiv empfundenen verminderten Schlafqualität und -menge im Menschen [2]. Erste, kleinere Studien mit dem Schlafhormon Melatonin in Alzheimer-Patienten sind erfolgt [3].

Methodik: Bei 26 gesunden älteren Probanden (75,1 \pm 3,5 Jahre) wurde die Abeta-Ablagerung im medialen prefrontalen Kortex (mPFC) mittels $\left[{ }^{11} \mathrm{C}\right] \mathrm{PIB}$ (Pittsburgh compound B) PET bestimmt. Zudem wurde ein fMRI (functional magnetic

Mander BA, Marks SM, Vogel JW et al. $\beta$-amyloid disrupts human NREM slow waves and related hippocampus-dependent memory consolidation. Nature Neuroscience 2015; 18: $1051-7$ resonance imaging) durchgeführt, um die Konsolidierung vor dem Nachtschlaf erlernter Wortpaare anhand der Hippokampusaktivität zu untersuchen. Der Schlaf erfolgte unter individuell gewohntem Schlaf-Wach-Habitus und wurde über acht Stunden polysomnografisch überwacht.

Ergebnisse: Der Anteil langsamer Hirnwellen im mPFC (0,6 - 1 $\mathrm{Hz}$ ) zeigte eine negative Assoziation mit der lokalen Abeta-Ablagerung, die selbst bei Korrektur für das Alter, das Volumen der mPFC grauen Substanz oder das Geschlecht signifikant blieb. Hierbei zeigte sich eine geminderte Anzahl langsamer Hirnwellen, aber keine auffällige Änderung in Steigung, Amplitude oder Dauer. Der Anteil der NREM-SWA war zudem positiv assoziiert mit der Gedächtnisleistung. Die Stärke der SWAAbnahme war mit der Fortdauer der Hippokampusaktivität korreliert: Die negative Assoziation zwischen dem Anteil der mPFC SWA und der linken Hippokampusaktivierung während erfolgreicher assoziativer episodischer Erinnerung war im fMRI größer als bei der korrekten Zurückweisung neuer Worte. Diese Assoziation war am stärksten im linken Hippokampus, trat jedoch bilateral auf. Anhand theoretischer Berechnungen (basierend auf root-mean-squared residual (RMR), goodness-of-fit index [GFI] und Bayesian information criterion [BIC]) ergab sich ein Modell, bei dem der Einfluss der fibrillären Abeta-Ablagerungen im mPFC auf die gestörte Erinnerungsleistung nicht direkt erfolgt, sondern durch die Schlafqualität vermittelt wird.

Schlussfolgerungen: Die lokale Abeta-Pathologie des mPFC ist assoziiert mit verminderten langsamen Hirnwellen des NichtREM-Schlafs, die wiederum ein Unvermögen der Hippokampusabhängigen Langzeit-Gedächtniskonsolidierung vorhersagen.

\section{- Kommentar von Kristina Endres, Mainz}

\section{Führt Schlafmangel zu Demenz?}

Die Autoren beschreiben einen Zusammenhang zwischen Abeta-Ablagerungen, Schlaf und Gedächtnisleistung. Wie sie selbst diskutieren, sind die Ergebnisse ihrer Studie jedoch querschnittsbasiert und korrelativer Natur. Die Bestätigung der hier gezeigten Daten in einer longitudinalen Studie wäre sicherlich wünschenswert. Da sowohl Schlaf als auch Gedächtnisleistung netzwerkabhängige Prozesse des Gehirns sind, können die linearen statistischen Modelle - basierend auf Veränderungen eines Hirnareals - tatsächliche Vorgänge vermutlich nur unzureichend abbilden. Sollten sich die Zusammenhänge in weiteren Studien bestätigen, wäre jedoch auch die Nutzung von Schlafqualitätskriterien als diagnostische Marker oder zur Erfolgskontrolle therapeutischer Interventionen denkbar. Zumindest wäre es interessant, eine mögliche Korrelation der Koinzidenz der Alzheimer-Demenz mit Schlafstörungen wie zum Beispiel bei Schichtdienstarbeitern oder Personen mit häufigem Jetlag zu untersuchen.

\author{
Referenzen: \\ Mander et al. Nat. Neurosci 2013 \\ . Spira et al. JAMA Neurol 2013 \\ 3. Cardinali et al. Curr Neuropharmacol 2010
}

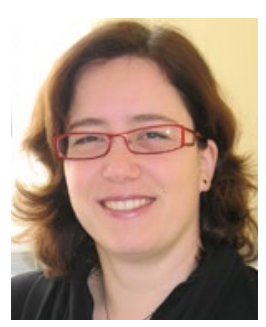

Dr. Kristina Endres, Mainz

Klinik für Psychiatrie und Psychotherapie Universitätsmedizin Johannes GutenbergUniversität Mainz

E-Mail: kristina.endres@unimedizin-mainz.de 